\title{
New Advances in Forming Functional Ceramics for Micro Devices
}

\author{
R.A. Dorey*, S. Rocks, F. Dauchy, A. Navarro \\ Cranfield University, Cranfield, Bedfordshire, MK43 OAL, UK \\ *r.a.dorey@cranfield.ac.uk, s.rocks@cranfield.ac.uk, f.dauchy.2003@cranfield.ac.uk, \\ a.navarro@cranfield.ac.uk
}

\begin{abstract}
Keywords: lead zirconate titanate, composite sol gel, thick film, micro-moulding, wet etching, MEMS
\end{abstract}

\begin{abstract}
Micro electromechanical systems (MEMS) are finding uses in an increasing number of diverse applications. Currently the fabrication techniques used to produce such MEMS devices are primarily based on 2-D processing of thin films. The challenges faced by producing more complex structures (e.g. high aspect ratio, spans, and multi-material structures) require the development of new processing techniques.

Potential solutions to these challenges based on low temperature processing of functional ceramics, selective chemical patterning, and micro-moulding are presented to show that it is possible to create complex functional ceramic structures which incorporate non-ceramic conducting and support structures. The capabilities of both techniques are compared and the relative advantages of each explored.
\end{abstract}

\section{Introduction}

Piezoelectric functional ceramics, such as lead zirconate titanate (PZT), find applications in a range of devices including ultrasound transducers, actuators, and sensors [1,2]. There exists a desire to reduce the size of these devices to increase performance, portability and applications. While large scale devices can be fabricated with ease using bulk materials that are shaped and assembled together, it becomes increasingly difficult to produce such structures at progressively smaller scales. To achieve the required reductions in size will require the use of microscale assembly techniques instead of traditional bulk processing routes. With microscale assembly techniques multiple materials are typically integrated and processed simultaneously. One of the primary issues that needs to be overcome when integrating ceramics with other materials typically employed in micro electromechanical systems (MEMS) (e.g. silicon, metals, glass) is the requirement for high temperatures to bring about densification of the ceramic material $[2,3]$.

The authors have combined multiple approaches for ceramic processing to develop a technique for producing structured thick $(10-50 \mu \mathrm{m})$ films. By combining low temperature sol gel processing with sintering aid assisted powder processing it has been possible to produce ceramic films with densities in the region of $80-95 \%$ theoretical density, at temperatures between 600 and $720^{\circ} \mathrm{C}$ [4]. Such low sintering temperatures are possible due to the use of the sol gel material which both increases the green density of the film beyond that achievable using powder processing alone, and fuses the powder particles together with a compositionally matched material. The incorporation of the small amount of sintering aid is then sufficient to still further increase the density of the film by $5-10 \%$ and consolidate the sol gel phase. Figure 1 shows an example of the cross-section of such a composite sol gel film showing the incorporation of a thin metallic electrode structure between two layers of ceramic. The figure demonstrates that it is possible to co-process ceramic and metallic materials on a silicon substrate. 


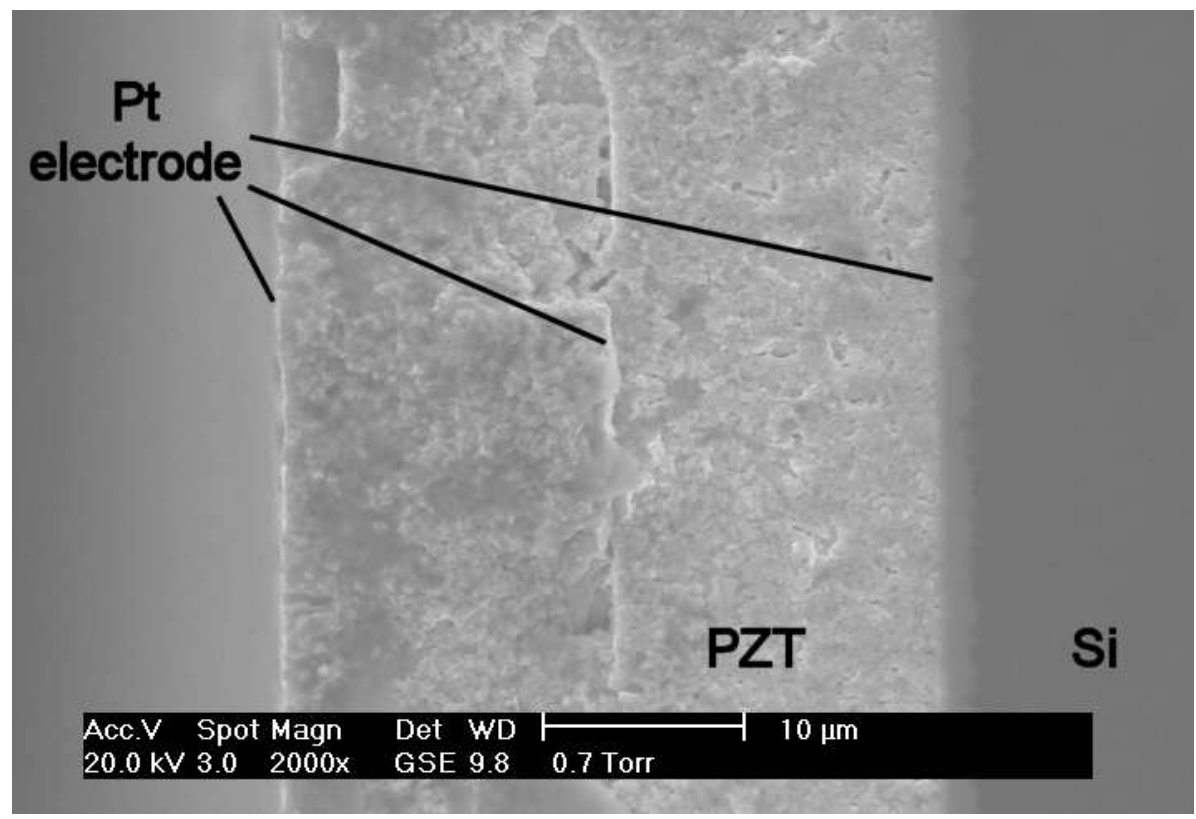

Fig. 1 - SEM photomicrograph showing a fracture cross section of a multilayer composite sol gel film, on a silicon substrate, incorporating multiple metal electrode layers each approximately

$100 \mathrm{~nm}$ thick.

The combined use of sol gel and powder processing has been demonstrated to be capable of realising integrated film structures with the potential to use such technology to produce functional integrated structures at low temperatures. The ability to integrate these materials to produce layered structures is, however, not sufficient to realise a functioning device. In order to produce a functioning device is also necessary to structure these films on a fine scale. This can be technically challenging due to the chemical inertness of many of these materials - especially ceramic materials. This paper will present and compare two approaches to structuring functional ceramics. The two approaches described are both based on the application of the composite sol gel technique, with either material removal or moulding being used to create structured features.

\section{Experimental}

Composite sol gel. The composite sol gel approach has been described in detail in previous publications [4]. Briefly, a sol is mixed with ceramic powder (and a sintering aid if required) to produce a slurry which can be spin coated onto a substrate. Table 1 gives details of the composition of the sol and suspension used for the two approaches. For the purposes of this comparison the slight differences in the compositions of the two systems can be ignored as they do not influence the processing conditions used.

Table 1 - Composition of the sol gel and slurry used

\begin{tabular}{|l|l|l|}
\hline & Wet etching [5] & Micro-moulding [6] \\
\hline Sol composition & $\begin{array}{l}\mathrm{Pb}\left(\mathrm{Zr}_{0.49} \mathrm{Ti}_{0.51}\right) \mathrm{O}_{3} \\
\text { lead acetate, zirconium isopropoxide, } \\
\text { titanium propoxide, } \\
\text { acetic acid/2-methoxyethanol solvent }\end{array}$ & $\begin{array}{l}\mathrm{Pb}\left(\mathrm{Zr}_{0.5} \mathrm{Ti}_{0.5}\right) \mathrm{O}_{3} \\
\text { lead acetate, zirconium isopropoxide, } \\
\text { titanium propoxide, } \\
\text { acetic acid/1-propanol solvent }\end{array}$ \\
\hline Slurry composition & $\begin{array}{l}1.5 \mathrm{~g} \text { of } \mathrm{PZ26} \text { powder (Ferroperm): } \\
1 \mathrm{ml} \text { sol }\end{array}$ & $\begin{array}{l}1.5 \mathrm{~g} \text { of } \mathrm{PZ26} \text { powder (Ferroperm): } \\
1 \mathrm{ml} \text { sol }\end{array}$ \\
& $\begin{array}{l}\mathrm{Cu} 2 \mathrm{O}+\mathrm{PbO} \text { (sintering aid) } \\
\mathrm{KR} 55 \text { (Dispersant- Kenrich } \\
\text { petrochemicals) }\end{array}$ & $\begin{array}{l}\text { KR55 (Dispersant- Kenrich } \\
\text { petrochemicals) }\end{array}$ \\
\hline
\end{tabular}


Wet etching. Continuous films were fabricated using the sol gel approach where the substrate was coated with the slurry which was then spun off at $2000 \mathrm{rpm}$ for 30 seconds to produce a uniform film approximately $2.5 \mu \mathrm{m}$ thick. The film was then dried at $200^{\circ} \mathrm{C}$ and pyrolysed at $450^{\circ} \mathrm{C}$ to convert the sol gel phase to an amorphous oxide ceramic. The density of the composite layer was then increased by infiltrating the layer with pure sol which was again spun at 2000rpm, dried and $200^{\circ} \mathrm{C}$ and pyrolysed at $450^{\circ} \mathrm{C}$. This process was repeated either 2,3 or 4 times to produce films of different densities. The film thickness was increased by depositing further composite/sol layers until the required film thickness had been achieved.

To structure the film a $5 \mu \mathrm{m}$ thick layer of photoresit (AZ4562) was deposited and selectively exposed to UV light, and developed to create a patterned mask on the surface of the PZT layer. A $\mathrm{HCl} / \mathrm{HF}$ etchant was then used to etch the exposed areas of PZT and in doing so produce structured PZT micro-features.

Micro-moulding. To produce the micro-moulds a thick layer of photoresit (AZ4562) was first deposited onto the surface of the substrate. The phororesit was then selectively exposed to UV and developed to create the micro-moulds. The micro-moulds were filled by coating the wafer with the composite slurry and spinning the substrate at 2000rpm to remove the excess. Instead of drying the composite slurry at $200^{\circ} \mathrm{C}$ the slurry was allowed to dry at room temperature prior to another composite layer being deposited. The high temperature drying stages were not conducted at this intermediate stage as they would have resulted in damage to the phororesist micro-moulds. Once the micro-moulds had been filled the wafer was dried and pyrolysed in a furnace using a ramp rate of $2^{\circ} \mathrm{C} / \mathrm{min}$ and a hold temperature of $600^{\circ} \mathrm{C}$ for 20 minutes. This drying/pyrolysis stage was conducted with the wafer suspended upside down to that any unwanted PZT would fall away from the sample and not contaminate the sample.

\section{Results}

Wet etching. Figure 3 shows an example of a structure realised using wet etching of a continuous film of PZT. During etching a non soluble product $(\mathrm{PbClF})$ is produced and remains as a white precipitate which slows the etching process $[5,7]$. For thin film processing this $\mathrm{PbClF}$ is typically removed using a $\mathrm{HNO}_{3}$ wash at the end of the etching process. The presence of this residue during etching does not result in a significant decrease in the etching rate of thin films as only a small amount of material is formed. For etching of thick film structures the presence of larger amounts of $\mathrm{PbClF}$ can cause a noticeable decrease in the etching rate. Unfortunately, it is not possible to remove this precipitate at regular intervals as the $\mathrm{HNO}_{3}$ wash also removes the protective photoresit from the PZT. Instead a final water wash, with ultrasonic agitation, is used to remove the $\mathrm{PbClF}$ product at the end of the etcing process.

The structure shown in figure 3 demonstrates the small structures can be produced using this etching process. The figure also clearly shows the characteristic side wall profile of the structures produced. These characteristic sloped side walls are a results of the isotropic nature of the etching process where material is removed at the same rate in the through-thickness direction as is removed in the in-plane direction. The uniform etch wall shown in figure 3 is characteristic of etching of an isotropic material. Figure 4 shows how the profile achieved can be radically altered if an anisotropic material is etched. In this case a larger number of intermediate sol infiltrations have been used with the result that an inhomogeneity is introduced at the surface of each composite layer. This subtle change in material properties (in particular etch characteristics) results in the through-thickness etch processes being momentarily slowed in relation to the in-plane etch process with the result that a stepped structure is obtained. 


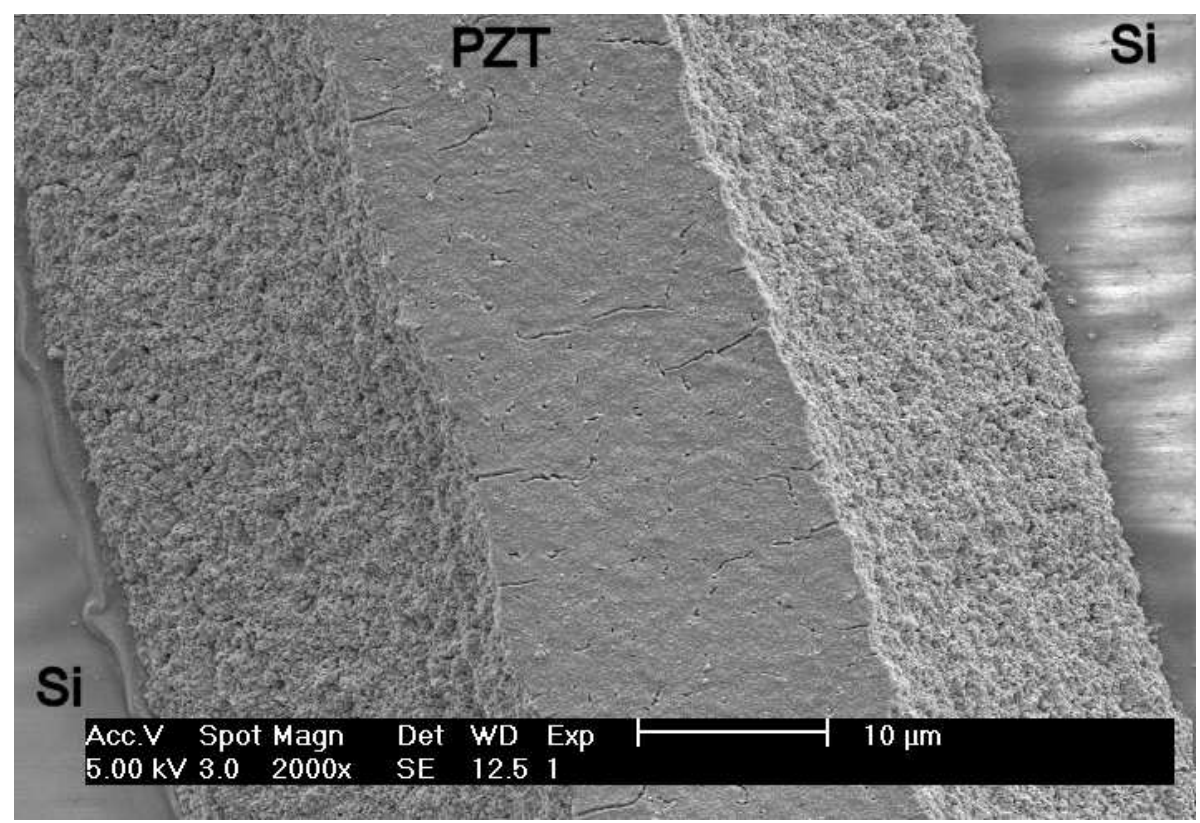

Fig. 3 - SEM photomicrograph of PZT structure produced by wet etching a homogenous thick $(\sim 21 \mu \mathrm{m})$ continuous film.

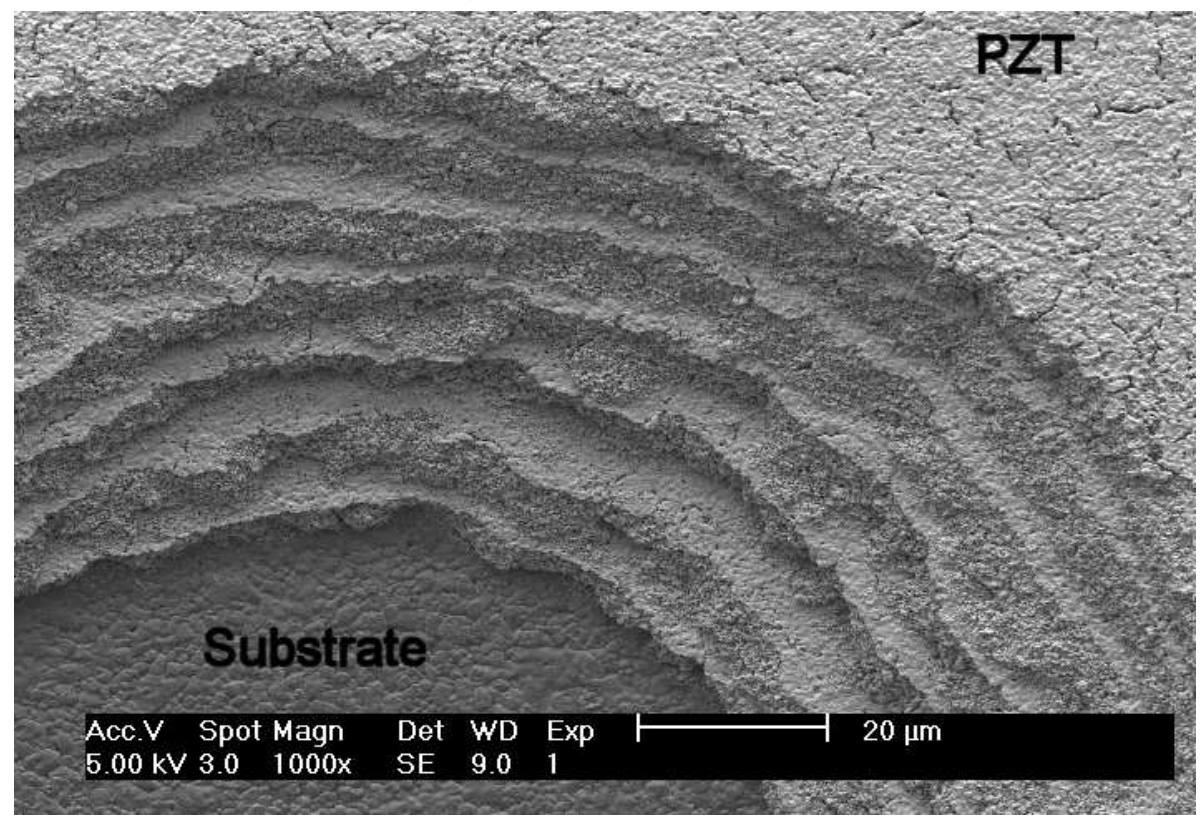

Fig. 4 - SEM photomicrograph of PZT structure produced by wet etching an inhomogenous thick $(\sim 40 \mu \mathrm{m})$ continuous film.

Micro-moulding. Structures of comparable size can also be produced using the micro-moulding technique as can be seen in figure 5. Using the micro-moulding process the shapes are formed directly within moulds. In comparison to the edge profile of the features produced using wet etching, the features produced using the micro-moulding technique exhibit more defined edge profiles.

Figure 5 also shows some of the defects that can occur when processing structures using the micromoulding technique. These defects occur when some of the ceramic material remains on top of the polymeric mould material. Where this occurs material may remain in place bridging two regions as can be seen between the two vertical lines in figure 5. Alternatively, such bridges may break away causing damage to the remaining structure as can be seen at the corners of the horizontal section. 


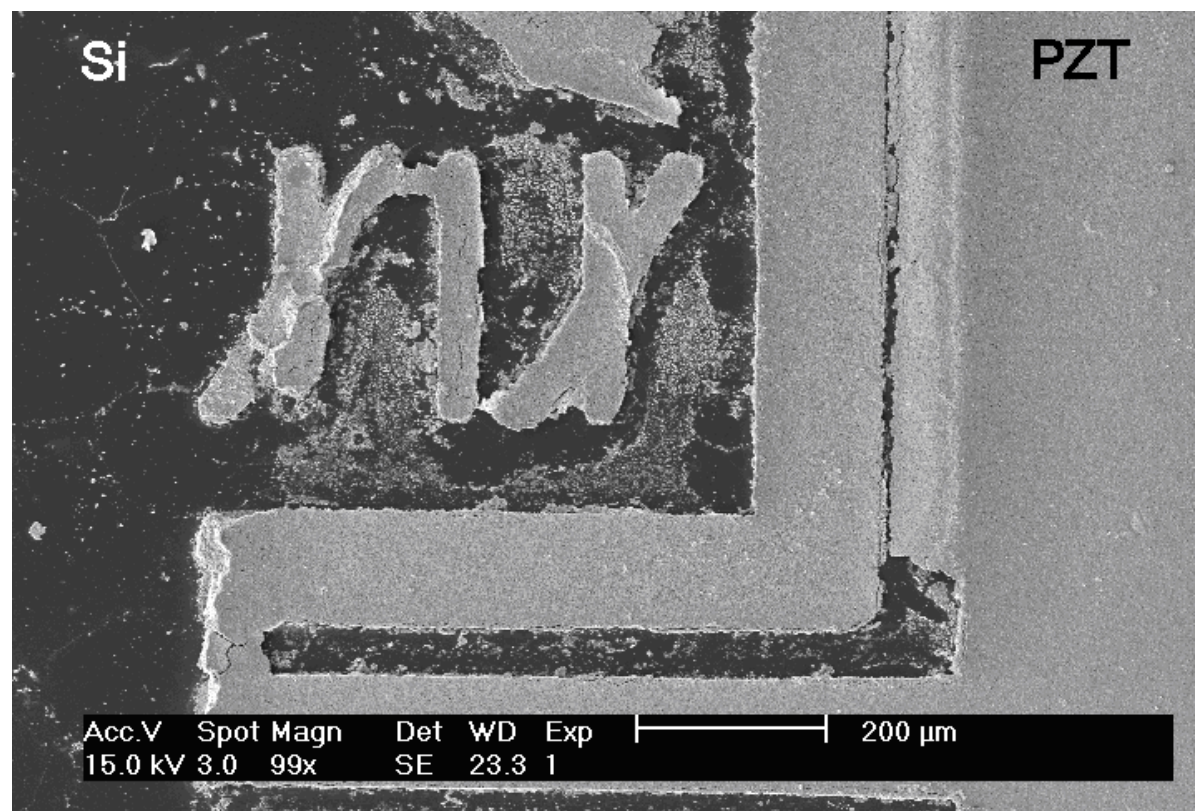

Fig. 5 - SEM photomicrograph of PZT structure produced using micro-moulding technique

\section{Comparison of shaping techniques}

Overview. For the purposes of comparing the two shaping techniques it is useful to consider the geometric requirements of a generic MEMS device. Typically the ability to produce small structures and to position them accurately and close to each other will be of interest. It is, therefore, appropriate to consider the minimum feature size achievable, accuracy and resolution as indicators of shaping capability. Table 2 and figure 6 provide a summary of the relative capabilities of the two techniques in relation to the 3 shaping indicators. 


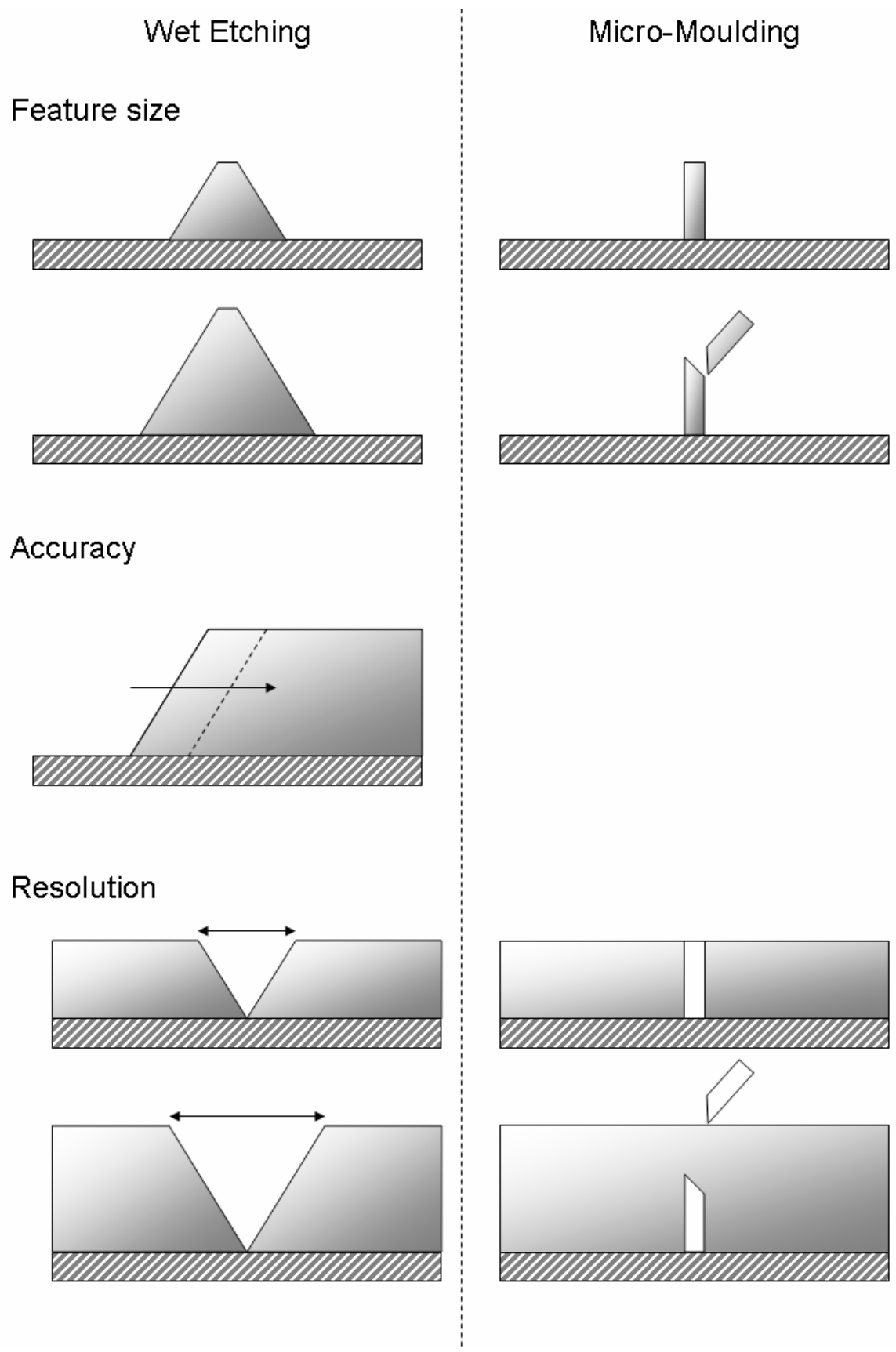

Fig 6. - Schematic representation of the relative limiting factors of the wet etching and micromoulding technuqes

Minimum feature size achievable. For the wet etching process the minimum feature size achievable is primarily limited by the ability to control the etching process. Provided this is controlled it is possible to produce features with very small size. It should be noted, however, that this small size will only relate to the top surface of the feature. As the features exhibit a marked side wall slope, the base of the feature will be significantly larger than the top. The ultimate extension of this would the production of micro-pyramids where the tip of the pyramid is a point and the base is between 2 and 3 times the film thickness across.

Accuracy. Both techniques use photolithograpy as a basis for positioning the features relative to each other. As this process is based on producing a chrome-glass shadow mask the positioning accuracy is dictated by the production of the shadow mask and subsequent photolithography process. In this respect the position of each feature relative to the other features is fixed by the quality of the mask produced. 
The exact control of the size of the final features is, however, subject to experimental variations. In the case of wet etching, the etching time will influence the amount of material removed so that shorter etch times will result in larger features while longer etch time will result in smaller features. For the micro-moulding process shrinkage/swelling of the photoresit moulds will affect the dimensions of the final features. This degree of shrinkage/swelling is much less than the size changes observed when etching times are varied. In this respect micro-moulding has the potential to provide greater accuracy in terms of final feature size.

Resolution. The resolution of the two processes are both dependent on the resolution of the photolithographic process as without being able to resolve individual features in the photoresit structure it will not be possible to create features. In addition to this criteria a second criteria based on the interactions of the film must also be considered. With wet etching the isotropic etch characteristic of the material imposes a far larger limitation on the resolution. As has been shown in figures 3 and 4, the resultant features exhibit a sloped side wall meaning that for a structure to be separated at the base the separation at the top will be related to the slope of the side wall angle. In practice this equates to a spacing of between 2 and 3 times the film thickness. Hence the resolution of the wet etching process is strongly dependent on the film thickness. When micro-moulding is used the resolution is dependent on the ability to create a self supporting photoresit 'wall' in the fist instance, and to remove the photoresit and any excess ceramic material remaining above the photoresit. In practice it is the ability to create the self supporting photoresit 'wall' which will limit the ultimate resolution of the process. This becomes particularly critical as the height of the mould cavities increase and the aspect ratio of these separating walls must increase.

Table 2 - comparison of capabilities of wet etching and micro-moulding in terms of minimum feature size, positioning accuracy and resolution

\begin{tabular}{|l|l|l|}
\hline Minimum feature size & Wet etching & Micro-moulding \\
\hline Accuracy & $\begin{array}{l}\text { Low angle side walls. } \\
\text { Minimum size of feature near } \\
\text { top surface controlled by } \\
\text { control of etching. Minimum } \\
\text { size of feature at base is a } \\
\text { function of etch wall angle and } \\
\text { film thickness. }\end{array}$ & $\begin{array}{l}\text { High angle side walls. } \\
\text { Minimum size of feature } \\
\text { related to ability to fill mould } \\
\text { and for structure to be self } \\
\text { supporting }\end{array}$ \\
\hline $\begin{array}{l}\text { Relative position of features to } \\
\text { each other defined by the } \\
\text { photolithographic process. } \\
\text { Size of final shape dictated by } \\
\text { under- or over-etch conditions. }\end{array}$ & $\begin{array}{l}\text { Relative position of features to } \\
\text { each other defined by the } \\
\text { photolithographic process. } \\
\text { Size of final shape dictated by } \\
\text { shrinkage/swelling of } \\
\text { photoresit mould and slurry } \\
\text { ink. }\end{array}$ \\
\hline Resolution & $\begin{array}{l}\text { Low angle side walls. } \\
\text { Proximity of two adjoining } \\
\text { features dictated by etch wall } \\
\text { angle and film thickness. }\end{array}$ & $\begin{array}{l}\text { Related to ability to create self } \\
\text { supporting photoresist wall and } \\
\text { successfully remove photoresist } \\
\text { and excess material after } \\
\text { moulding process. }\end{array}$ \\
\hline
\end{tabular}

Summary. Both the micro-moulding and the wet etching technique can be used to produce a range of shaped functional ceramic features for integration into MEMS devices. Micro-moulding allows structures with high side wall angles to be fabricated which could be advantageous for smaller 
structures where deviations from 'ideal' shapes may cause problems. However, with larger area structures such slight changes in the shape would not be expected to have significant effects on the performance of any devices produced.

When considering micro-moulding the height of the feature is ultimately controlled by the thickness of the photoresist that can be deposited and patterned. The photoresist used in this study (AZ4562) is suitable for creating micro-moulds a few 10's of micrometres high using standard UV lithography techniques. However, to create much deeper moulds it would be necessary to use an alternative photoresit (e.g. SU8) which may be significantly more difficult to remove without damaging the ceramic structures produced.

\section{Conclusions}

Two techniques for shaping functional ceramics for use in integrated micro devices have been described. The techniques, based on low temperature thick film processing, make use of selective material removal and micro-moulding to create features with heights (or thicknesses) of a few micrometres to a few 10's of micrometres and widths/lengths ranging from a few micrometres upwards.

Each technique has relative advantages and disadvantages which limit the resolution, accuracy or minimum features size. In general, micro-moulding is most suitable for situations where small features with high angle walls are required, while wet etching is more applicable to larger features where side wall definition is not as critical. However, used as part of suite of techniques, both approached offer potential to create a new generation of integrated functional devices combining a range of materials including silicon, ceramics, metals and glasses.

\section{References}

[1] N. Setter: J.Euro.Ceram.Soc. Vol. 21 (2001), pp1279-1293.

[2] R.A. Dorey, R.W. Whatmore: J. Electroceramics, Vol. 12 (2004), pp19-32.

[3] S.M. Spearing: Acta mater. Vol. 48 (2000), pp179-196.

[4] R.A. Dorey, S.B. Stringfellow, R. W. Whatmore: J.Euro.Ceram.Soc. Vol. 22 (2002), pp29212926

[5] F. Dauchy, R.A. Dorey: Proceedings of the 4M conference 2005 (Elsevier).

[6] A. Navarro, S.A. Rocks, R.A. Dorey: to be published in J. Electroceramics.

[7] K. Zheng, J. Lu, J. Chu: Microprocesses and Nanotechnology Conference International 2003

Digest of Papers Vol. 29-31 (2003), pp 248-249 